\title{
Visualization of Hydrogen on Ti-6Al-4V Using Hydrogen Microscope
}

\author{
Kei Tanaka ${ }^{1}$, Shinichiro Kato ${ }^{2}$, Masahiro Kitaura ${ }^{1}$ and Kazuyuki Ueda ${ }^{3}$ \\ ${ }^{1}$ Daido Bunseki Research Inc., Nagoya 457-8545, Japan \\ ${ }^{2}$ Daido Steel Co., Ltd., Nagoya 457-8545, Japan \\ ${ }^{3}$ Nano High Tech Research Center, Toyota Technological Institute, Nagoya 468-8511, Japan
}

\begin{abstract}
The two-dimensional distribution of hydrogen on Ti-6Al-4V gives very important information on the environmental embrittlement mechanism. The scanning-electron-stimulated desorption ion microscope, the so-called hydrogen microscope, can be used to visualize hydrogen on solid surfaces. In this study, we attempt to visualize hydrogen on Ti-6Al-4V alloy using a hydrogen microscope. As a result, the hydrogen distribution on the Ti-6Al-4V surface was clearly observed. [doi:10.2320/matertrans.M2009410]
\end{abstract}

(Received December 11, 2009; Accepted April 21, 2010; Published June 2, 2010)

Keywords: titanium-aluminum-vanadium alloy, hydrogen embrittlement, hydrogen visualization, microscopy

\section{Introduction}

Ti-6Al-4V alloy is widely used in the aerospace, chemical, medical and automotive industries because of its high strength/gravity ratio and resistance to corrosion. However, it is well known that this alloy has the severe problem of hydrogen embrittlement. ${ }^{1,2)}$ Therefore, it is very important to determine the hydrogen-trapping sites in the material. Nevertheless, the investigation of hydrogen-trapping sites has been a pending issue, because the visualization of hydrogen is very difficult.

Some techniques for the visualization of hydrogen have been achieved. The hydrogen microscope developed by Ueda et $a .^{3)}$ can be used to observe hydrogen directly on solid surfaces, and moreover, can measure the two-dimensional (2D) hydrogen distribution by adopting the time-of-flight electron-stimulated desorption (TOF-ESD) technique. Novel 2D hydrogen images for various solid surfaces have been published. ${ }^{4-7)}$ In this study, we successfully attempted to visualize hydrogen on Ti-6Al-4V using a hydrogen microscope.

\section{Experimental}

The hydrogen microscope apparatus was custom-fabricated and consists of two electron guns, a microchannel plate detector and a three-grid mesh system, all of which are contained in an ultrahigh-vacuum system, as described in Ref. 3) in detail. The specimen was irradiated by a $900 \mathrm{eV}$ pulsed electron beam with a specimen bias of $+15 \mathrm{~V}$. To obtain a $2 \mathrm{D} \mathrm{H}^{+}$image, the electron beam was scanned digitally on the surface.

The material used for this study was Ti-6Al-4V alloy, which was provided by Daido Steel Co., Ltd., that had been heat-treated at a temperature above the beta transus at $1050^{\circ} \mathrm{C}$ for $1 \mathrm{~h}$, followed by cooling in air. Prior to observation, the specimen surface was cleaned by repeated Ar ion bombardment and annealing.

The total content of hydrogen solved in the material was measured by the inert gas fusion thermal conductivity detection method (Horiba, EMGA621A). To enable metal-

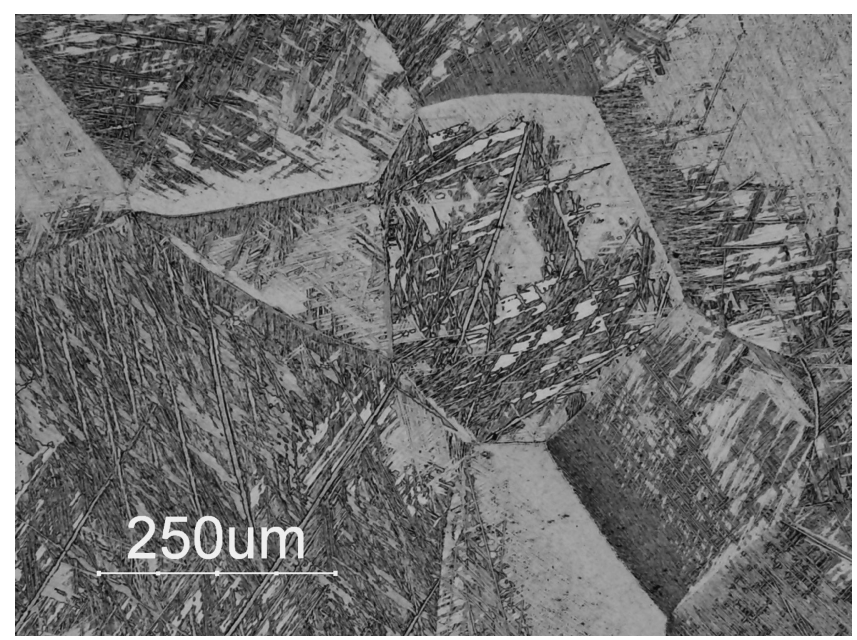

Fig. 1 Microstructure of Ti-6Al-4V obtained by optical microscopy.

lographic analysis by optical microscopy, a specimen was prepared by mechanical polishing followed by chemical etching. To identify the phases, X-ray diffraction (XRD, Rigaku, RINT TTR3) analysis was performed. Electron probe microanalysis (EPMA, JEOL, JXA-8900RL) was also performed to determine the chemical composition.

\section{Results and Discussion}

The microstructure of Ti-6Al-4V alloy has an acicular $\alpha+$ $\beta$ structure, as shown in Fig. 1, and the average colony size was found to be several hundred micrometers. The initial content of gaseous hydrogen in this material is $57 \mathrm{ppm}$. An XRD pattern of the specimen is shown in Fig. 2, from which the hexagonal (hcp) $\alpha$ and the cubic (bcc) $\beta$ phases were detected; however, the hydride and oxide patterns could not be confirmed.

Figure 3 shows a typical TOF spectrum for Ti-6Al-4V. In the TOF spectrum "P" denotes a soft X-ray signal generated by an electron impact. The peak corresponding to the hydrogen ion $\left(\mathrm{H}^{+}\right)$appeared at approximately $2.5 \mu \mathrm{s}$. Using this peak, the resulting $\mathrm{H}^{+}$image is illustrated in Fig. 4, in 


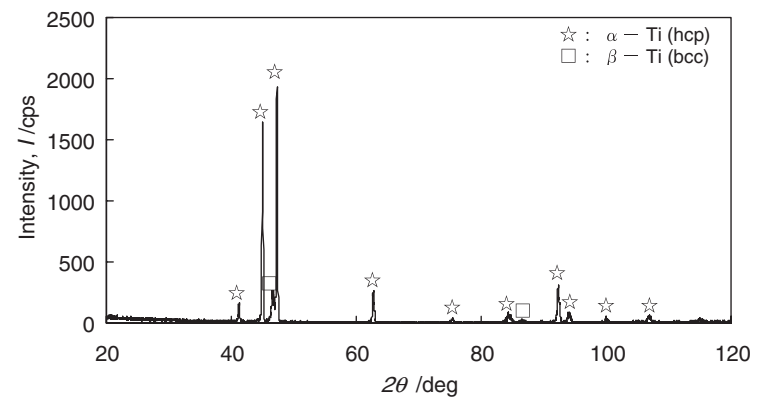

Fig. 2 XRD pattern of Ti-6Al-4V.

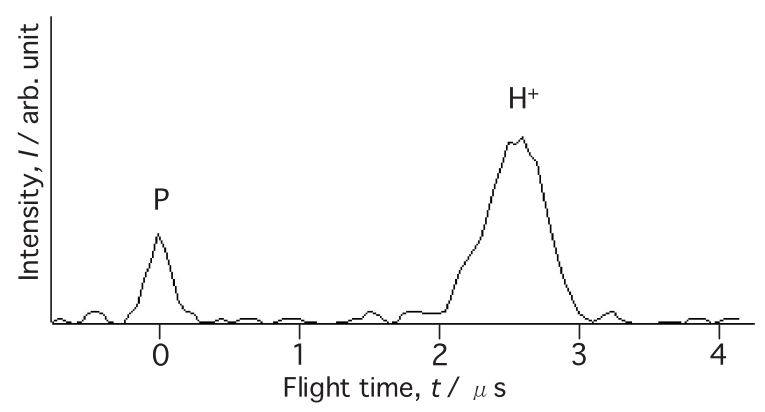

Fig. 3 Typical TOF spectrum for Ti-6Al-4V.

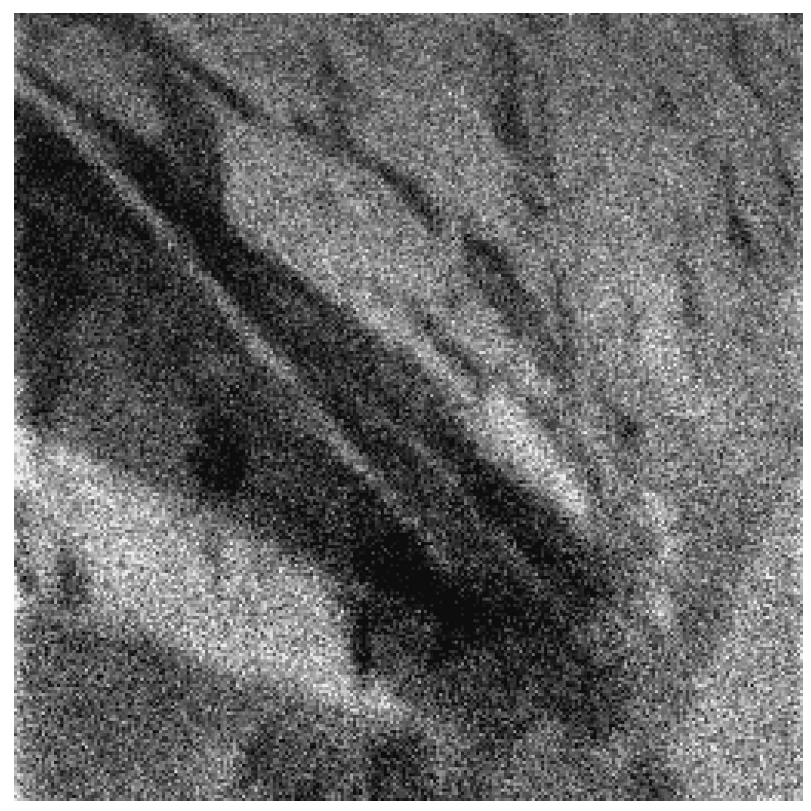

Fig. 4 Image of $\mathrm{H}^{+}$on Ti-6Al-4V obtained by hydrogen microscopy.

which the hydrogen distribution is clearly observed. The white area corresponds to a high concentration of hydrogen on the material.

A backscattering electron (BSE) image of the same area as that in Fig. 4 was obtained by EPMA as shown in Fig. 5. In the BSE image, dark and bright areas are observed. The contrast between them is shown in Fig. 6, where the hatched areas indicate the dark areas in Fig. 5. Comparing the images in Fig. 4 and Fig. 6, it is clarified that many hydrogen atoms exist in the dark area.

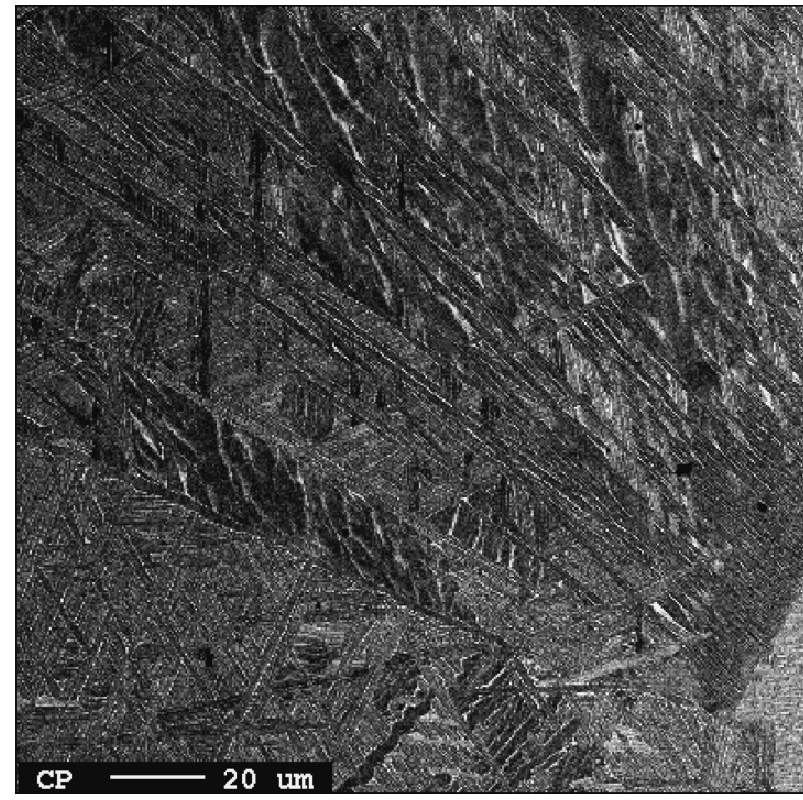

Fig. 5 BSE image of Ti-6Al-4V obtained by EPMA.

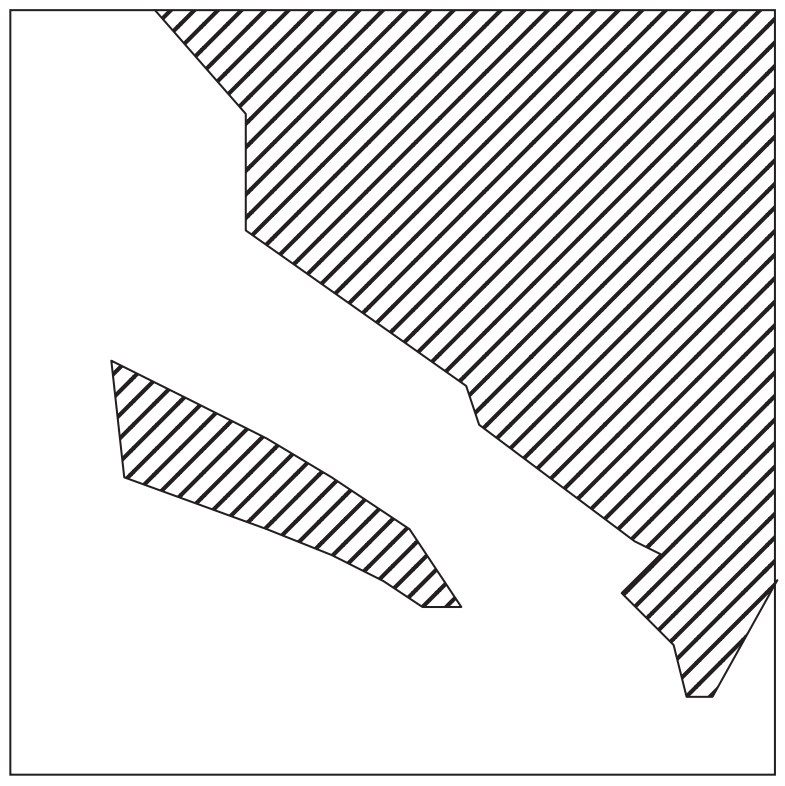

Fig. 6 Hatched areas corresponding to dark areas in the BSE image.

It is known that the contrast in a BSE image is due to the chemical composition and/or crystal structure. We consider that the hydrogen concentration is related to the crystal structure. The chemical composition is almost the same in the dark and bright areas, as confirmed by EPMA. In the BSE image, the $\alpha$ phase is observed as the dark area because of the channeling contrast caused by the difference in the crystal structure, i.e., $\alpha$ and $\beta$ phases. Therefore, it was found that many hydrogen atoms exist in the $\alpha$-phase region. However, the detailed relationship between the hydrogen concentration and the crystal structure will be clarified by analysis of the electron backscatter diffraction pattern (EBSD). 


\section{Conclusion}

The hydrogen distribution on Ti-6Al-4V alloy was visualized using a hydrogen microscope. In the observed distribution of hydrogen, a relationship between the hydrogen concentration and the contrast in BSE images was recognized.

\section{Acknowledgements}

This work was supported by the "High-Tech Research Center" project for Private Universities: matching fund subsidy from the Ministry of Education, Culture, Sports, Science and Technology (MEXT), 2001-2008. The authors thank Dr. Koyanagi of Daido Steel Co., Ltd., for providing the Ti-6Al-4V specimen, and Dr. Ishikawa of Daido Bunseki Research Inc., for his helpful discussions.

\section{REFERENCES}

1) R. D. Daniel, R. J. Quigg and A. R. Troiano: Trans. ASM 51 (1959) 843861.

2) R. R. Boyer and W. F. Spurr: Met. Trans. A 9 (1978) 23-29.

3) K. Ueda, K. Ishikawa and M. Yoshimura: Jpn. J. Appl. Phys. 36 (1997) L1254-L1256.

4) K. Ueda, K. Ogai, K. Ishikawa and M. Yoshimura: Jpn. J. Appl. Phys. 39 (2000) 4363-4365.

5) K. Ueda, K. Ishikawa, K. Ogai and M. Yoshimura: Jpn. J. Appl. Phys. 39 (2000) L315-L317.

6) K. Ueda, K. Ishikawa and K. Ogai: Surf. Sci. 493 (2001) 138-142.

7) K. Ueda and Y. Itoh: Surf. Sci. 600 (2006) 1684-1688. 